\title{
CHARACTERIZATION OF FUNCTIONALIZED COMPOSITE SWCNT FOR SENSING APPLICATION.
}

\section{Yawar Alamgeer Research Scholar, Department of Physics, L. N. Mithila University, Darbhanga}

ABSTRACT It has been seen that an individual carbon nanotube can be bent and tangled within a bundle, increasing the difficulty of exfoliation and debundling of SWCNTs aggregates. As the surface area of untangled SWCNTs are quite high as compared to those exist in bundles, efforts have been made to debundle the SWCNTs and make the use of its unique properties. Increase in surface area increases the sensing ability of the CNTs. Since the most common gas sensing principle is the adsorption and desorption of gas molecules on sensing materials, it is quite understandable that by increasing the contact interfaces between the analytes and sensing materials, the sensitivity can be significantly enhanced. Functionalization can improve the sensitivity and selectivity of the CNTs based gas sensors. Chemical bonds might be used to facilitate the interaction of the nanotube with other analytes such as solvents, polymers and biopolymers matrices. The adsorption of these molecules on the nanotubes is associated with a partial charge transfer, who alters the charge-carrier concentration as the electronic properties of semiconducting CNTs are very sensitive to certain analytes. After interaction with gas molecules and charge transfer, the resulting change in the electrical resistance of the nanotube is utilized as a sensor signal. Functionalization of CNTs with PANI makes it selective towards NH3 gas, with PEI makes it selective towards NO2 gas and HFIP derivatives makes it selective towards DMMP gas. Thus functionalization can provide a better pathway for the CNTs for utilization in potential applications. Further characterization using FTIR and Raman of the given samples were done to ensure the potential of the samples as gas sensor.

\section{KEYWORDS :}

Experimental Details:

A requisite amount of carboxylated SWCNT was taken in a bottle and dissolved in $50 \mathrm{ml}$ DI water. Now the prepared sample was ultrasonicated by bath sonicator (BlackstoneNEY-Ultrasonics) for one and half hour between $40 \mathrm{kHz}$ to 80 $\mathrm{kHz}$. After ultrasonication, appropriate amount of aqueous solution of PEI was added to the dispersed solution. Now the dispersed solution was kept under stirring for approximately 24 hours. The resultant solution was again ultrasonicated for approximately $\mathrm{l} \mathrm{hr}$. and then filtered over $0.2 \mu \mathrm{m}$ polycarbonate millipore membrane through vacuum filtration pump to obtain desired composite. The sample was taken for FTIR and Raman analysis. The description of the samples which were prepared is shown in table 1.0 :

TABLE 1.0:

\begin{tabular}{|c|c|c|}
\hline S.No. & Description of the Sample & $\begin{array}{c}\text { Composition of the } \\
\text { Sample (PEI:CNT) }\end{array}$ \\
\hline 1. & SWNT-COOH + PEI (DI water) & PEI:CNT $=1: 10$ \\
\hline 2. & SWNT-COOH + PEI (DI water) & PEI:CNT = 1 $: 5$ \\
\hline 3. & SWNT-COOH + PEI (DI water) & PEI:CNT $=1: 4$ \\
\hline 4. & SWNT-COOH + PEI (DI water) & PEI:CNT $=1: 3.33$ \\
\hline 5. & SWNT-COOH + PEI (DI water) & PEI:CNT $=1: 2.5$ \\
\hline 6. & SWNT-COOH + PEI (DI water) & PEI:CNT $=1: 2$ \\
\hline
\end{tabular}

Characterization of given samples-

\section{Fourier Transform Infrared Spectroscopy:}

FTIR spectroscopy was used to monitor both molecular interactions and concentration of volatile organic chemicals adsorbed onto CNTs surface. These includes minimal preparation, the ability to use neat samples, observation of useful overtone and combination bands, and that most bands were visible in a single FTIR spectrum without sample dilution or spectral scaling. For FTIR measurements, the specimens were submitted with a powder, using $\mathrm{KBr}$ as background. In order to eliminate the influence of water and other contaminations, both the $\mathrm{KBr}$ and the sample tablets were pretreated in-situ with temperature of $80{ }^{\circ} \mathrm{C}$ for $2 \mathrm{~h}$. The background of $\mathrm{KBr}$ and the atmosphere in the cell were deduced. All the spectra baselines were corrected. The measurements were done using FTIR spectrometer (Varian680-IR spectrometer) The FTIR spectrum of SWCNT-COOH, $\mathrm{PEI}$ and their composite are shown from figure 1.0 to figure 1.4 :

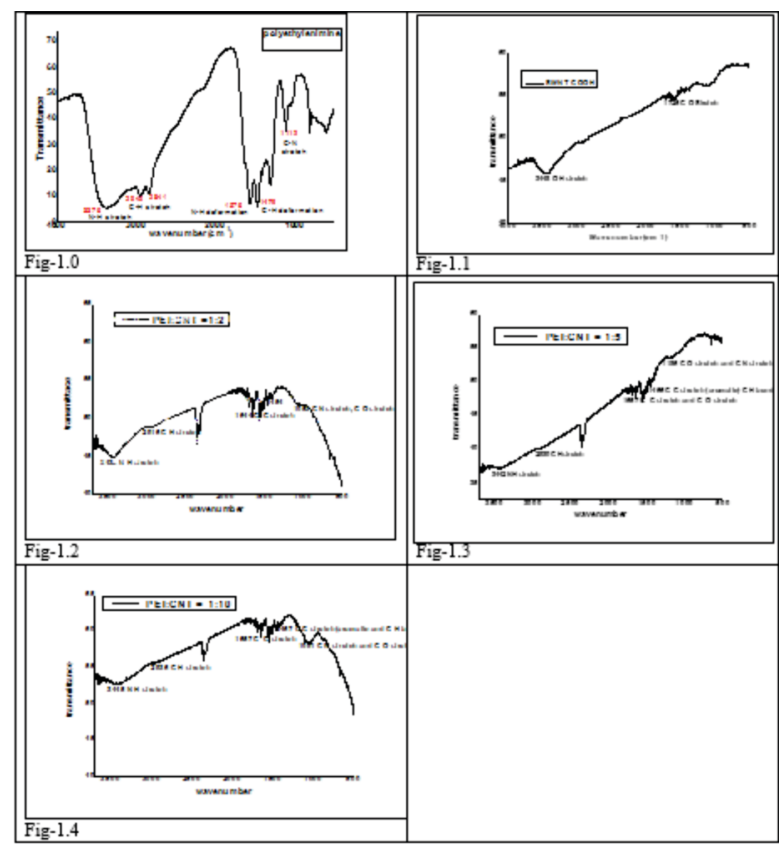

FTIR Spectrum of Fig.1.0-PEI,Fig.1.1-SWNT-COOH,Fig.1.2composite having PEI: CNT=1:2,Fig.1.3- the sample having PEI: CNT = 1: 5 and Fig.1.4-the sample having PEI: CNT =1: 10

The IR spectra of SWCNT-COOH+PEI composite shows the bands at $1737 \mathrm{~cm}^{-1}$ related to $\mathrm{C}=\mathrm{O}$ Stretch which indicates the presence of carboxyl group. At $1650 \mathrm{~cm}^{-1}$, peak of $\mathrm{C}=\mathrm{C}$ stretch is observed which indicates the presence of carbon bonds of SWCNTs as well as indicates the $\mathrm{N}-\mathrm{H}$ bend due to the presence of amine group in PEI. At around $2920 \mathrm{~cm}^{-1}$ peak of C$\mathrm{H}$ Stretch is observed due to presence of aliphatic chain in PEI. At $3440 \mathrm{~cm}^{-1}$, peak of N-H stretch indicates the presence of amine group due to PEI. Peaks of $\mathrm{C}-\mathrm{H}$ deformation at nearly $1460 \mathrm{~cm}^{-1}$ and C-N stretch at nearly $1066 \mathrm{~cm}^{-1}$ and $1186 \mathrm{~cm}^{-1}$ were also observed. Peak at $1737 \mathrm{~cm}^{-1}$ are due to carboxyl group of SWCNT-COOH.

The FTIR spectroscopy was carried out for the samples to confirm the functionalization of SWCNTs-polymer composites 
obtained. From the spectra it can be concluded that noncovalent functionalization has taken place between carboxyl SWCNTs and PEI as no new bond formation has taken place. Non-covalent functionalization occurs simply by the adsorption of polymer over the surface of the material. Some of the PEI got adsorbed onto CNT surface via electrostatic charge interaction between $\mathrm{NH}^{+}$of PEI and $\mathrm{COO}$ of carboxylic group of SWCNT.

For covalent functionalization to take place, formation of some new bonds must be evident. In the case of carboxyl SWCNT-PEI composite, covalent functionalization must take place via amide bond formation via nucleophilic substitution where nitrogen donates its lone pair to the carboxyl carbon atom of SWCNTs. As in the spectra, no evidence of amide bond has been observed so there must be very feeble chances of covalent functionalization.

\section{Raman Spectroscopy:}

Raman Spectroscopy is frequently employed to study the quality of the Carbon Nanotubes. It also provides information about the number of the walls, and the presence of crystalline or amorphous carbon, including the diameter of SWCNT. As the D-band is the diagnostic of disruptions in the hexagonal framework of the SWCNTs, the variation of the relative intensity of the $\mathrm{D}$ band to the $\mathrm{G}$ band can provide direct evidence for the covalent modification of SWCNTs.

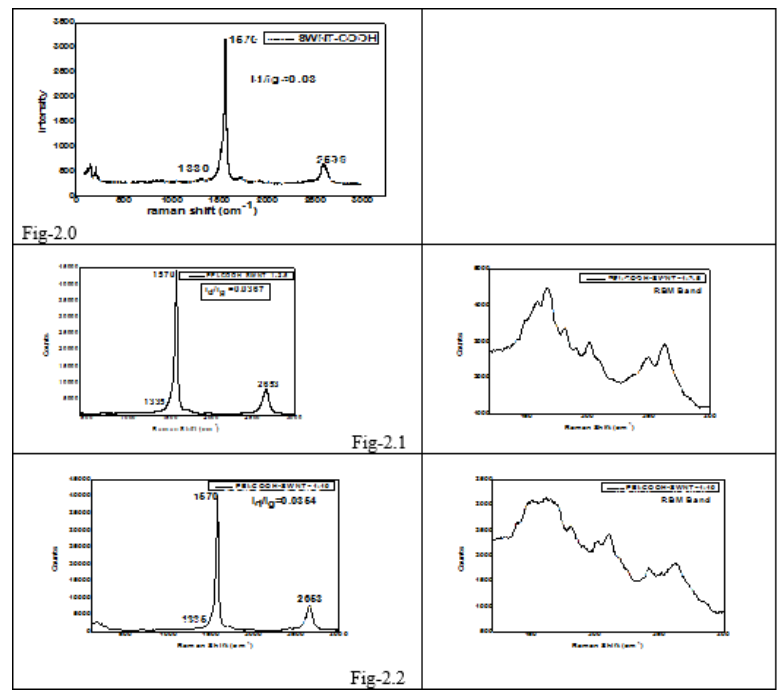

Raman Spectra of Fig. 2.0 -SWCNT-COOH,Fig.2.1Functionalized PEI Composite (PEI: SWCNT-COOH =1:2.5) and Fig.2.2-Functionalized PEI Composite (PEI: SWCNT$\mathrm{COOH}=1: 10$ )

The non-covalent attachment can be also confirmed by Raman spectroscopy (Fig. 2.0 and 2.2). Spectra of SWCNTsPEI composites having concentration (PEI: SWCNT$\mathrm{COOH}=1: 2.5$ and $1: 10$ ) after functionalization contained the following characteristic peaks: the D band located at $1335 \mathrm{~cm}^{-1}$ which is corresponds to the $\mathrm{sp}_{3}$ hybridized carbon atoms in rings which are correlated with the extent of nanotubes sidewalls defects and chemical sidewall functionalization and the $\mathrm{G}$ band centered at $1575 \mathrm{~cm}^{-1}$ which arises due to bond stretching of all pairs of $\mathrm{sp}_{2}$ atoms in both rings and chains. The multiple peaks observed in the radial breathing mode (RBM) of SWCNTs could be ascribed to a distribution of diameters in the SWNT samples. From the RBM band, the diameter of SWCNTs used were calculated by the equation:

$\omega_{R B M}\left(\mathrm{~cm}^{-1}\right)=\frac{224\left(\mathrm{~cm}^{-1}\right)}{d_{0}(\mathrm{~nm})}$

Thus the diameter of carboxylated SWCNTs used in our work ranges from $0.8 \mathrm{~nm}$ to $1.2 \mathrm{~nm}$. No variation in $\mathrm{Id} \backslash \mathrm{Ig}$ ratio of the composite means defects were not increased after the functionalization. This confirms that there is non-covalent attachment of PEI on surface of carboxylated SWCNT. Thus non-covalent attachment can also be confirmed by Raman spectroscopy. From the above figures it can be clearly seen that the nanotubes of different chiralities are present in the carboxylated SWCNTs used in making composite as many peaks arises in the RBM band of the composites. These peaks refer to the different diameter of the nanotubes corresponding to which one or more chirality may be present.

After functionalization, G band and the D band shifted from their position and located at $1570 \mathrm{~cm}^{-1}$ and $D$ band at $1335 \mathrm{~cm}^{-1}$ respectively. $G^{\prime}$ band is also shifted from its position and located at $2653 \mathrm{~cm}^{-1}$. This shows that non covalent functionalization has taken place. In Raman spectra G'band in PEI-SWCNT has a higher Raman shift by $54 \mathrm{~cm}^{-1}$ compared to that of carboxylated SWCNTs. On functionalization of CNTs, heavy atoms get attached to the CNTs so this causes change in the vibrations between the bonds and hence there is upshift of the band. The id/ig ratio in SWCNT-COOH is 0.03 and in composite id/ig ratio is also nearly 0.03 . Thus the Raman results also confirm the non covalent type of functionalization between PEI and carboxylated SWCNT.

\section{CONCLUSION-}

The FTIR spectroscopy was carried out for the samples to confirm the functionalization of SWCNTs-polymer composites obtained. From the spectra it can be concluded that noncovalent functionalization has taken place between carboxyl SWCNTs and PEI as no new bond formation has taken place. Non-covalent functionalization occurs simply by the adsorption of polymer over the surface of the material. Some of the PEI got adsorbed onto CNT surface via electrostatic charge interaction between $\mathrm{NH}^{+}$of $\mathrm{PEI}$ and $\mathrm{COO}^{-}$of carboxylic group of SWCNT.

For covalent functionalization to take place, formation of some new bonds must be evident. In the case of carboxyl SWCNT -PEI composite, covalent functionalization must take place via amide bond formation via nucleophilic substitution where nitrogen donates its lone pair to the carboxyl carbon atom of SWCNTs. As in the spectra, no evidence of amide bond has been observed so there must be very feeble chances of covalent functionalization.

The non-covalent attachment can be also confirmed by Raman spectroscopy.Spectra of SWCNTs-PEI composites having concentration (PEI: SWCNT-COOH=1:2.5 and 1:10) after functionalization contained the following characteristic peaks: the $\mathrm{D}$ band located at $1335 \mathrm{~cm}^{-1}$ which is corresponds to the $\mathrm{sp}_{3}$ hybridized carbon atoms in rings which are correlated with the extent of nanotubes sidewalls defects and chemical sidewall functionalization and the $\mathrm{G}$ band centered at 1575 $\mathrm{cm}^{-1}$ which arises due to bond stretching of all pairs of $\mathrm{sp}_{2}$ atoms in both rings and chains. The multiple peaks observed in the radial breathing mode (RBM) of SWCNTs could be ascribed to a distribution of diameters in the SWCNT samples.

Thus the diameter of carboxylated SWCNTs used in our work ranges from $0.8 \mathrm{~nm}$ to $1.2 \mathrm{~nm}$. No variation in Id $\backslash \mathrm{Ig}$ ratio of the composite means defects were not increased after the functionalization. This confirms that there is non-covalent attachment of PEI on surface of carboxylated SWCNT. The $\mathrm{id} / \mathrm{ig}$ ratio in SWCNT-COOH is 0.03 and in composite id/ig ratio is also nearly 0.03 . Thus the Raman results also confirm the non covalent type of functionalization between PEI and carboxylated SWCNT.

\section{REFERENCES:}

1. Hirsch, A., Angewandte Chemie-International Edition 41 (11), 1853-1859 (2002).

2. Banerjee, S., Hemraj-Benny, T., and Wong, S. S., Advanced Materials 17 (1), 
17-29 (2005).

3. Gogotsi, Y., ed., Carbon Nanomaterials. (Taylor and Francis Group, LLC, Boca Raton, London, New York, 2006.

4. Azamian, B. R., Coleman, K. S., Davis, J. J., Hanson, N., and Green, M. L. H., Chemical Communications (4), 366-367 (2002)

5. Hamon, M. A., Chen, J., Hu, H., Chen, Y. S., Itkis, M. E., Rao, A. M., Eklund, P. C., and Haddon, R. C., Advanced Materials 11 (10), 834-+ (1999).

6. Kuznetsova, A., Popova, I., Yates, J. T., Bronikowski, M. J., Huffman, C. B., Liu, J., Smalley, R. E., Hwu, H. H., and Chen, J. G. G., NEXAFS and vibrational spectroscopic studies, Journal of the American Chemical Society 123 (43), 10699-10704 (2001)

7. Klumpp, C., Kostarelos, K., Prato, M., and Bianco, A., , Biochimica Et Biophysica Acta-Biomembranes 1758 (3), 404-412 (2006).

8. Ramanathan, T., Fisher, F. T., Ruoff, R. S., and Brinson, L. C., Chemistry of Materials 17 (6), 1290-1295 (2005)

9. Hamwi, A., Alvergnat, H., Bonnamy, S., and Beguin, F., Carbon 35 (6), 723-728 (1997).

10. Okotrub, A.V. Yudanov, N. F, Chuvilin, A. L., Asanov, I. P, Shubin, Y. V. Bulusheva, L. G., Gusel'nikov, A.V., and Fyodorov, I. S., Chemical Physics Letters 322 (3-4), 231-236(2000)

11. Touhara, H. and Okino, F., Property control of carbon materials by fluorination, Carbon 38 (2), 241-267 (2000)

12. Hamon, M. A., Hui, H., Bhowmik, P., Itkis, H. M. E., and Haddon, R. C., Applied Physics a-Materials Science \& Processing 74 (3), 333-338 (2002).

13. Chen, J., Hamon, M.A., Hu, H., Chen, Y. S., Rao, A. M., Eklund, P. C., and Haddon, R. C Science 282 (5386), 95-98 (1998).

14. Dyke, C. A. and Tour, J. M., Journal of the American Chemical Society 125 (5), 1156-1157 (2003).

15. Balasubramanian K. and Burghard M, Small l (2), 180-192 (2005).

16. Kong J, Franklin NR, Zhou CW, Chapline MG, Peng S, Cho KJ,Dai HJ (2000) Science 287:622.

17. Li J, Lu Y, Ye Q, Cinke M, Han J, Meyyappan M (2003) NanoLett 3:929. Doi: $10.1021 / \mathrm{nl} 034220 \mathrm{x}$

18. Neamen, D.A. Semiconductor Physics and Devices. Richard D. Irwin: Homewood, IL, 1992.

19. On the production of $\alpha$ blue substance by the electrolysis of sulphate of aniline H. Letheby Journal of the Chemical Society Volume 15, 161-163, 1862doi:10.1039/JS8621500161. 